\title{
Services as Parameter to Provide Best QOS : AN ANALYSIS OVER WIMAX
}

\author{
Kuldeep Singh $^{1}$ and Reena Sharma ${ }^{2}$ \\ ${ }^{1}$ Department of Electronics \& Communication Engineering,GCET,Greater Noida, India \\ nitkuldeep@gmail.com \\ ${ }^{2}$ Department of Electrical \& Electronics Engineering, GCET, Greater Noida, India \\ 174 reena@gmail.com
}

\begin{abstract}
In this paper it is proposed to provide the QoS to the user by using the degradation of service under hostile environment being itself be a parameter to improve the QoS. Here the relation between the service and environment of its best performance drawn on the basis of simulation and analysis.The service then taken as a parameter to decide present environment of the user and to take measurable steps to improve the QoS either doing handover to nearby station or increasing power or to provide some marginal bandwidth etc.All analysis done over a WiMax network i.e. being designed and simulated using the Qualnet wireless simulator.
\end{abstract}

\section{KEYWORDS}

WiMax, Qualnet, USG, ertPS, rtPS, nrtPS, BE

\section{INTRODUCTION}

QoS means the offer versus delivery of a service. A quality of service framework is a fundamental component of a $4 \mathrm{G}$ broadband wireless network for satisfactory service delivery of evolving multimedia applications to end users, and managing the network resources.

In recent years, telecommunication operators are constantly seeking more efficient wireless broadband service, while telecommunication technology is continuously upgrading its access network technologies to cope with the high demands for high-speed internet access and multimedia service by end-users.

It is still a big challenge to maintain QoS providing AAA. Unlike wireless LANs, WiMAX networks incorporate several quality of service (QoS) mechanisms at the Media Access Control (MAC) level for guaranteed services for data, voice and video.The bandwidth is allocated to mission-critical, time-critical application without affecting any of the other applications or nodes in the network. WiMAX seems to be the solution as it is able to provide easy deployment, high speed data rate and wide range coverage. Most importantly, WiMAX provides Quality of Service (QoS) that can support all kinds of real-time application in wireless networks that includes priority scheduling and queuing for bandwidth allocation that is based on traffic scheduling algorithms within wireless networks. [3] 
After going through the Wimax QoS structure in section 2.The relation between the service and environment of its best performance being drawn on the basis of simulation and analysis in section 5.Finally its concluded that how the QoS can be provide to the user by using the degradation of service under hostile environment being itself be a parameter to improve the QoS.

\section{WIMAX QOS STRUCTURE}

The MAC layer of 802.16 (WiMAX) is designed to differentiate service among traffic categories with different multimedia requirements. The five data delivery service classes are

Unsolicited Grant Service (USG): UGS supports real-time service flows that generate a fixedsize data packet on a periodic basis, e.g., VoIP without silence suppression.

Extended Real-time Polling Service (ertPS): ertPS supports features of UGS with variable-size data packets, such as Voice over IP with silence suppression.

Real-Time Polling Service (rtPS): rtPS supports real-time service flows that generate variablesize data packets on a periodic basis, such as MPEG video or Voice over IP with silence suppression.

Non-real-time Polling Service (nrtPS): nrtPS supports delay-tolerant data streams consisting of variable-sized data packets for which a minimum data rate is required, such as FTP or HTTP (web browsing).

Best Effort (BE): BE service supports data streams for which no minimum service level is required and which may therefore be handled on a space-available basis. such as the transmission of HTTP[11]

Table 1. Comparison of WiMax QoS Classes [5]

\begin{tabular}{|l|l|l|}
\hline \multicolumn{1}{|c|}{ Q ${ }_{0 S}$} & \multicolumn{1}{|c|}{ Pros } & \multicolumn{1}{c|}{ Cons } \\
\hline UGS & $\begin{array}{l}\text { No overhead. Meet guaranteed latency for real-time } \\
\text { service }\end{array}$ & $\begin{array}{l}\text { Bandwidth may not be utilized fully since allocations } \\
\text { are granted regardless of current need. }\end{array}$ \\
\hline ertPS & Optimal latency and data overhead efficiency & $\begin{array}{l}\text { Need to use the polling mechanism (to meet the delay } \\
\text { guarantee) and a mechanism to let the BS know when } \\
\text { the traffic starts during the silent period. }\end{array}$ \\
\hline rtPS & Optimal data transport efficiency & $\begin{array}{l}\text { Require the overhead of bandwidth request and the } \\
\text { polling latency (to meet the delay guarantee) }\end{array}$ \\
\hline nrtPS & $\begin{array}{l}\text { Provide efficient service for non-real-time traffic with } \\
\text { minimum reserved rate }\end{array}$ & N/A \\
\hline BE & $\begin{array}{l}\text { Provide efficient service for BE traffic } \\
\text { Nong service guarantee; some connections may starve for } \\
\text { long period of time. }\end{array}$ \\
\hline
\end{tabular}

\section{AdvanTAges OF QOS IN WiMAX}

Low latency for mission-critical and mission-critical applications

Data prioritization (Bandwidth management)

Optimized transmission of multimedia applications . 


\section{RELATED WORK}

For assuring best QoS i.e. to achieve $\mathrm{ABC}$ (always best connected), a lot of work has been done for the area such as

2006 : Claudio Cicconetti, Luciano Lenzini, and Enzo Mingozzi, Carl Eklund proposed that it is possible to employ scheduling algorithms that have been proposed for wired environments, which are able to provide QoS guarantees. In simulations, they have evaluated the deficit round robin(DRR) and weighted round robin (WRR )scheduling algorithms as possible candidates for algorithms to be implemented in a production system.[9]

2008 : HY Tung, KF Tsang, KT Ko verified that the QoS aware bandwidth allocation with threshold setting lowers the Blocking Probability of rtPS and nrtPS. [8]

2009 : Chakchai So-In, Raj Jain, and Abdel-Karim Tamimi classified recent scheduling disciplines based on the channel awareness in making the decision. Well-known scheduling discipline can be applied for each class such as EDF for rtPS and WFQ for nrtPS and WRR for inter-class. With the awareness of channel condition and with knowledge of applications, schedulers can maximize the system throughput or support more users.[5]

2011 : Rakesh Kumar Jha, Upena D Dalal, Suresh Limkar, Bholebawa Idris Zoherbhai in their networks model observed that there are a lot of factor involved i.e. distance between node (Fixed and Mobile) from BS altitude and LOS is different in different scenario with client with location with respect to BS. We also observed that QoS has effected with different Application (Here Voice over IP Telephony and MPEG).[3]

2012 : Here in this paper we proposed that as there is storng relationship between the envoirnm,ent and the Services, we can using services itself as an approach to improve QoS.

\section{SimUlation ENVIRONMENT}

Qualnet, is a wireless network simulator in which using the IP protocols precedence field, applications can direct traffic to a specific service class. Table specifies the mapping between precedence values and service classes[13].This is the parameter being used to decide the service.

\subsection{MAC layer Service Flow Mapping}

Table 2. MAC Layer Service Flow Mapping

\begin{tabular}{|c|c|}
\hline IIAC Layer Services & Precedence \\
\hline Unsolicited Grant Service & 7,5 \\
\hline Extended real-time polling Service & 4 \\
\hline Real-time polling Service & 3 \\
\hline Non-Real-time polling Service & $6,2,1$ \\
\hline Best Effort & 0 \\
\hline
\end{tabular}




\subsection{Simulation Configuration}

Rest of the settings are

\begin{tabular}{|c|c|}
\hline CHANNEL FREQUENCY & $2.4 \mathrm{GHz}$ to $2.46 \mathrm{G}$ \\
\hline PROPAGATION MODEL : & STATISTICAL \\
\hline PROPAGATION LIMIT: & $-111.0 \mathrm{dBM}$ \\
\hline SHADOWING MODEL: & CONSTANT \\
\hline PATHLOSS MODEL : & $*$ \\
\hline ENVOIRNMENT & $*$ \\
\hline PATHLOSS MODEL : & $*$ \\
\hline FADING MODEL: & $*$ \\
\hline SIMULATION TIME : & $150 \mathrm{Sec}$ \\
\hline PHYSICAL TEMPERATURE: & $290.0 \mathrm{~K}$ \\
\hline PHYSICAL NOISE FACTOR : & 10.0 \\
\hline ANTENNA MODEL : & Omni Directional \\
\hline ANTENNA GAIN : & $12.0 \mathrm{DB}$ \\
\hline ANTENNA HEIGHT : & 10.0 Meters \\
\hline
\end{tabular}

* Signifying The Parameters changed Each Time To Set Different Environments.

CBR : SOURCE: NODE 16 DESTINATION: NODE 15

INTERVAL: 0.2 SEC

\subsection{Simulation Result and Description}

Observation with accurate value

Table 3. Environment vs. Precedence(Accurate)

\begin{tabular}{|c|c|c|c|c|c|c|c|c|}
\hline $\begin{array}{c}\text { Environment } \\
\text { Precedence }\end{array}$ & & Env l & Env 2 & Env 3 & Env 4 & Env 5 & Env6 & Env7 \\
\hline \multirow{3}{*}{0} & Throughput & 166242 & 23704 & 35189 & 25998 & 10676 & 0 & 10286 \\
\cline { 2 - 10 } & Delay & 0.155312129 & 0.045536037 & 6.507874546 & 0.509012904 & 0.054538592 & 0 & 0.028184648 \\
\cline { 2 - 10 } & Jitter & 0.014306039 & 0.018228657 & 0.103889034 & 0.044179832 & 0.167555150 & 0 & 0.011589889 \\
\hline \multirow{3}{*}{2} & Throughput & 150142 & 9584 & 36799 & 14305 & 7443 & 0 & 10423 \\
\cline { 2 - 10 } & Delay & 0.188677898 & 0.610726465 & 1.511074263 & 3.185379053 & 0.146059036 & 0 & 0.027801640 \\
\cline { 2 - 10 } & Jitter & 0.015109532 & 0.0312498552 & 0.181527787 & 0.076557627 & 0.021802409 & 0 & 0.011870787 \\
\hline \multirow{3}{*}{3} & Throughput & 142476 & 0 & 29197 & 9181 & 2349 & 0 & 3078 \\
\cline { 2 - 10 } & Delay & 4.109533089 & 0 & 2.276087619 & 0.474973108 & 1.775009781 & 0 & 0.457413434 \\
\cline { 2 - 10 } & Jitter & 0.022190664 & 0 & 0.198017515 & 0.019279418 & 0.488396558 & 0 & 0.025072371 \\
\hline \multirow{4}{*}{4} & Throughput & 183989 & 0 & 34312 & 17792 & 31438 & 0 & 11803 \\
\cline { 2 - 10 } & Delay & 0.039104983 & 0 & 3.058028446 & 0.027104184 & M0.490616113 & 0 & 0.001820496 \\
\cline { 2 - 10 } & Jitter & 0.001623666 & 0 & 0.179544696 & 0.002763757 & 0.021913191 & 0 & 0.001820496 \\
\hline \multirow{3}{*}{5} & Throughput & 183930 & 0 & 34312 & 14766 & 21088 & 0 & 11803 \\
\cline { 2 - 9 } & Delay & 0.050735121 & 0 & 3.058028446 & 0.044999229 & 0.093866524 & 0 & 0.457413434 \\
\cline { 2 - 9 } & Jitter & 0.001602932 & 0 & 0.179544696 & 0.006634014 & 0.027590741 & 0 & 0.001820496 \\
\hline
\end{tabular}


Observation with range specification

Table 4. Environment vs. Precedence(Range)

\begin{tabular}{|c|l|c|c|c|c|c|c|c|}
\hline $\begin{array}{c}\text { Environment } \\
\text { Precedence }\end{array}$ & & Env 1 & Env 2 & Env 3 & Env 4 & Env 5 & Env 6 & Env7 \\
\hline \multirow{3}{*}{0} & Throughput & UM & H & UM & H & M & L & H \\
\cline { 2 - 9 } & Delay & M & L & H & M & L & L & L \\
\cline { 2 - 9 } & Jitter & M & M & L & UM & M & L & L \\
\hline \multirow{3}{*}{2} & Throughput & M & UM & H & LM & LM & L & UM \\
\cline { 2 - 9 } & Delay & M & L & L & H & M & L & L \\
\cline { 2 - 9 } & Jitter & M & H & M & H & L & L & M \\
\hline \multirow{3}{*}{3} & Throughput & L & L ( 0) & L & L & L & L ( 0) & L \\
\cline { 2 - 9 } & Delay & H & L & M & M & H & L & L \\
\cline { 2 - 9 } & Jitter & H & L & H & M & H & L & H \\
\hline \multirow{3}{*}{4} & Throughput & M & L & UM & UM & H & L & UM \\
\cline { 2 - 9 } & Delay & L & L & UM & L & M & L & L \\
\cline { 2 - 9 } & Jitter & L & L & M & L & L & L & L \\
\hline \multirow{3}{*}{5} & Throughput & H & L & UM & M & UM & L & H \\
\cline { 2 - 9 } & Delay & L & L & UM & L & L & L & H \\
\cline { 2 - 9 } & Jitter & L & L & M & L & L & L & L \\
\hline
\end{tabular}

H: HIGH

UM: UPPER MEDIUM

M: MEDIUM

L: LOWER

\subsection{Best Environment per Service}

The best environment per service concluded from Table 3. and Table 4. is as follow

Table 5. Best Environment per Service

\begin{tabular}{|c|c|c|}
\hline PRECEDENCE & SERVICE & ENVIORNMENT NO. \\
\hline 0 & Best effort & 3 \\
\hline 2 & Non-real-time Polling Service & 4 \\
\hline 3 & Real-time Polling Service & 4 \\
\hline 4 & Extended real-time Polling Service & 1 \\
\hline 5 & Unsolicited Grant Service & \\
\hline
\end{tabular}


International Journal of Next-Generation Networks (IJNGN) Vol.4, No.1, March 2012

\section{Matlab concluding Plots based on Observation}

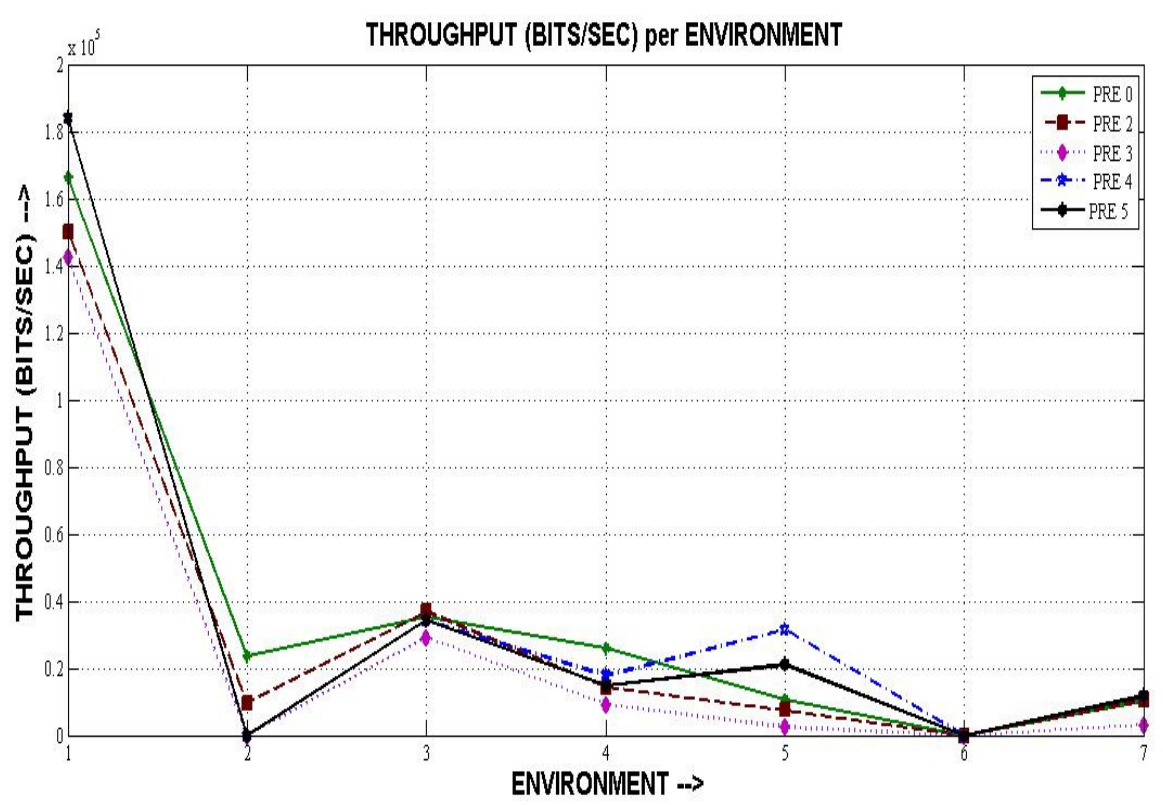

Figure 1. Throughput (Bits/Sec)

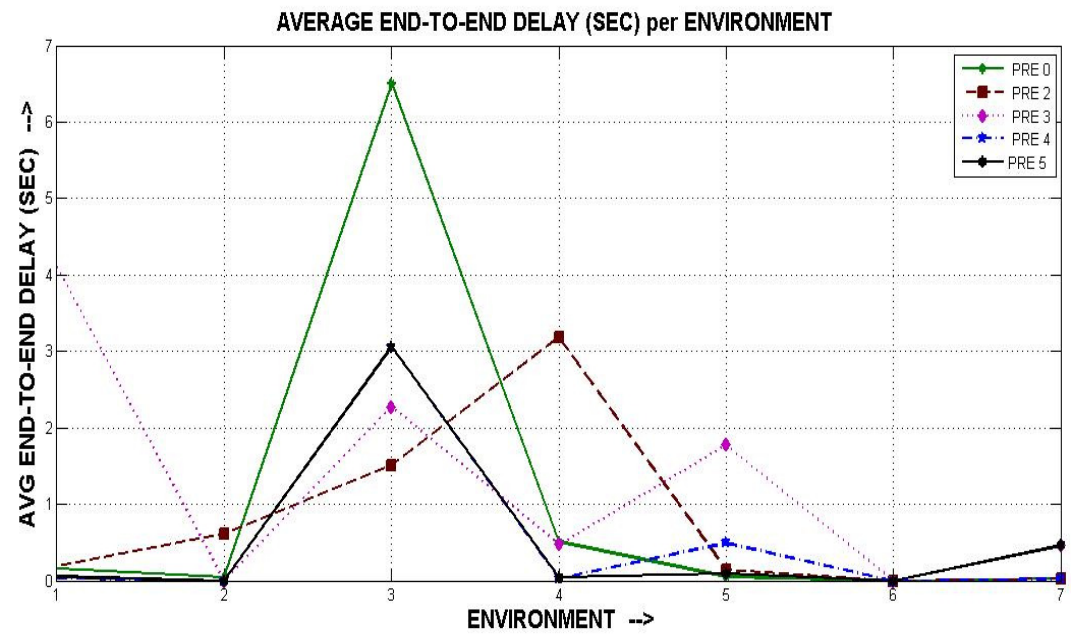

Figure 2. Average End-to-End Delay (Sec) 


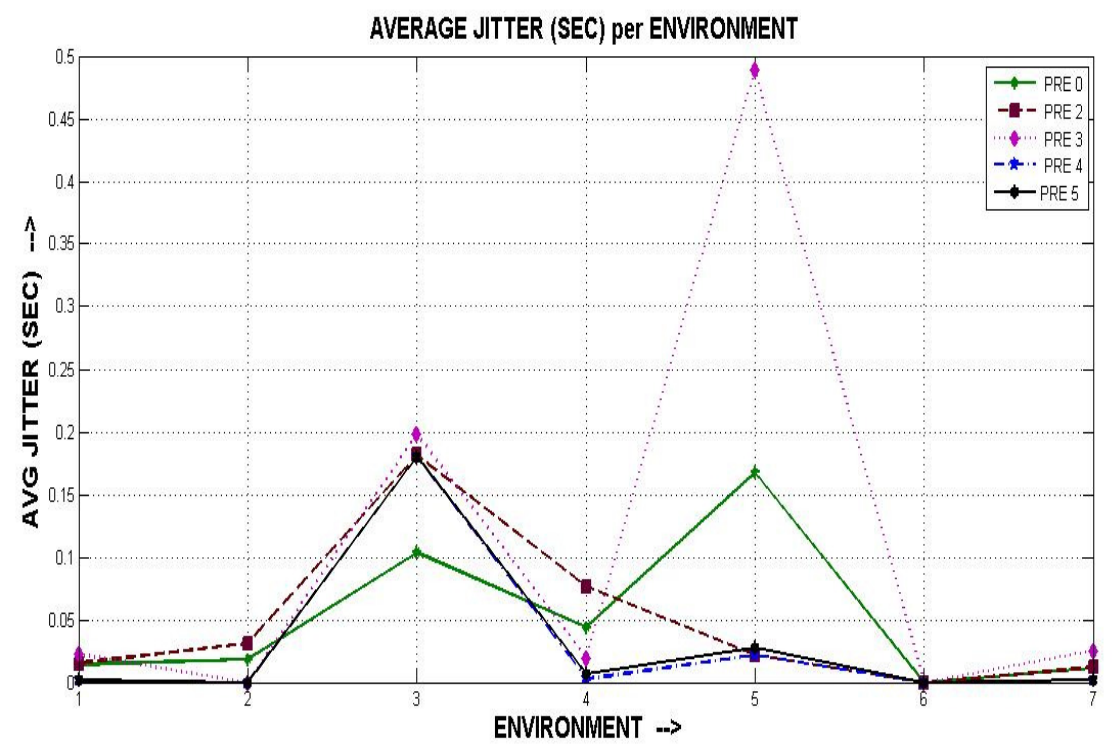

\section{ANALYSIS}

Figure 3. Average Jitter (Sec)

1.Best Effort service with precedence 0 provides best performance when the user switches from STREET M-to-M pathloss model to COST 231 HATA pathloss model.

2.Non-real-time Polling Service provides best performance when the user switches from TWO RAY pathloss model to semi urban area with STREET M-TO-M pathloss model.

3.Real-time Polling Service provide best performance when the user switches from semi urban area with STREET M-to-M pathloss model to OKUMARA HATA.

4.Extended real-time Polling Service provides best performance when the user switches from semi urban areas with STREET M-to-M pathloss model to OKUMARA HATA.

5.Unsolicited Grant Service provide best performance when the user switches from TWO RAY pathloss model to URBAN pathloss model

\section{CONCLuSion}

Due to fast development of technology, future communication and transmission are totally depends upon wireless network but quality of service $(\mathrm{QoS})$ provision is a bigger challenge for wireless communications.

The design constraints at several layers of the IEEE 802.11 restrict its capacity to deliver guaranteed QoS. Recently due to its large coverage area, low cost of deployment and high speed data rates,WiMAX emerges as a promising technology for providing wireless last mile connectivity.

In this paper we are given concept of WiMAX (Worldwide Interoperability for Microwave Access) network performance for QoS monitoring and optimization solution as Physical and MAC layer of this technology which defines 5 different data delivery service classes that can be 
used in order to satisfy Quality of Service (QoS) requirements of different applications, such as VoIP, videoconference, FTP, Web, etc.

With aims to provide always the best quality of service (QoS) for users. Both the performance of applications and network conditions are considered and we observed that each service that is provided to the user does not work well in every environment. Different services have their best performance under different environment as listed and concluded above under analysis part. The QoS for the particular service will become poor as the user switch over to more hostile environment.Thus degradation in particular service will give information about the surrounding and proper measures can be taken to improve Qos at base station or by the service provider.

\section{REFERENCES}

[1] Dong Ma, Student Member, IEEE, and Maode Ma, Senior Member, IEEE, “A QoS Oriented Vertical Handoff Scheme for WiMAX/WLAN Overlay Networks" , IEEE TRANSACTIONS ON PARALLEL AND DISTRIBUTED SYSTEMS, VOL. 23, NO. 4, APRIL 2012 ,pp 598-606.

[2] Sunil Kr. Singh, Ajay Kumar, Siddharth Gupta, Ratnakar Madan, "Architectural Performance of WiMAX over WiFi with Reliable QoS over Wireless Communication", IJANA Vol. 03, Issue: 01 Pages:1017-1024 (2011).

[3] Jha, Dalal, Limkar, Zoherbhai, "Performance of Location Based WiMAX Network for QoS with Optimal Base stations (BS)”, PROCEEDINGS OF ICETECT 2011,pp 857-863.

[4] Alasti , Neekzad, Hui and Vannithamby, Intel Labs , "Quality of Service in WiMAX and LTE Networks”, IEEE Communications Magazine • May 2010, pp 104-111 .

[5] Chakchai So-In, Raj Jain, and Abdel-Karim Tamimi, "Scheduling in IEEE 802.16e Mobile WiMAX Networks: Key Issues and a Survey", IEEE JOURNAL ON SELECTED AREAS IN COMMUNICATIONS, VOL. 27, NO. 2, FEBRUARY 2009, pp 156 - 171 .

[6] Liao, "Advanced seamless vertical handoff architecture for WiMAX and WiFi heterogeneous networks with QoS guarantees”,Elsevier , Computer Communications 32, 281-293. 2009.

[7] Rohit A. Talwalkar, Mohammad Ilyas, Motorola Inc, 8000 West Sunrise Blvd, "Analysis of Quality of Service (QoS) in WiMAX networks”, 978-1-4244-3805-1/08/\$25.00 -008IEEE.2008.

[8] HY Tung, KF Tsang, KT Ko , "QoS for Mobile WiMAX Networ ks: Call Admission Control and Bandwidth Allocation”, IEEE CCNC 2008 proceedings , pp 576 -580.

[9] Cicconetti, Lenzini, Mingozzi, Eklund, "Quality of Service Support in IEEE 802.16 Networks" , IEEE Network • March/April 2006 ,pp 50-55 .

[12] Jen--Chu Liu, "QoS Provisioning in WiMAXNetworks:Chances\&Challenges". Aug 2005. MNET Lab, CS, NTHU

[13] Michael Welzl, Leopold Franzens University of Innsbruck Max Mühlhäuser, Darmstadt University of Technology, "Scalability and Quality of Service: A Trade-off?", IEEE Communications Magazine ,32-36. June 2003.

[14] “QualNet Programmers Guide”, Scalable Network Technologies.

[15] http://degas.cis.udel.edu/QualNet

[16] http://www.scalable-networks.com 


\section{Authors}

Kuldeep Singh received his B.Tech. degree in Electronics \& Communication Engineering from Institute of Engineering \& Technology, CSJM University Kanpur.(Year: 2001-05) and M.Tech. degree in Electronics \& Communication Engineering from National Institute of Technology, Kurukshetra, Haryana (Year: 2007-09). He is currently working as an Assistant Professor in Electronics and Communication Engineering Department at Galgotias College of Engineering and Technology, Greateer Noida,U.P., India ,affiliated to GBTU, Lucknow, India. He has 4.5 years of teaching experience in Engineering College. His area of interest includes Wireless Networks

Reena Sharma received her A.M.I.E (ECE) degree from Institution of Engineers ,Kolkata (India) in 2008 and M.Tech. degree in Electronics \& Communication Engineering from National Institute of Technology, Kurukshetra, Haryana (Year: 2008-10). She is currently working as an Assistant Professor in Electronics and Electrical Engineering Department at Galgotias College of Engineering and Technology,Greateer Noida ,U.P., India ,affiliated to GBTU, Lucknow,India . She has 5 years experience in Engineering field and 2 years of teaching experience in Engineering College. Her area of interest includes Wireless Networks.
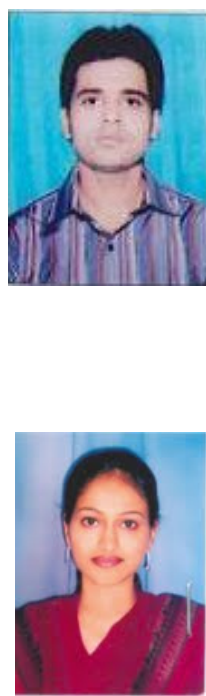